\title{
Uso da Netnografia para a Geração de Personas e Requisitos para Sistemas com foco em pessoas com Transtorno do Espectro Autista: Um Relato de Experiência
}

\author{
Anna Beatriz Marques, Gabriel Aires Melo \\ Universidade Federal do Ceará (Campus de Russas) - Russas, CE - Brasil \\ beatriz.marques@ufc.br, airesgabrielmelo15@gmail.com
}

\begin{abstract}
The early stages of software development require a good understanding of the target audience and their needs. Conventional methods used to achieve these goals are costly and researchers are subject to the interest of the target audience. This article reports the application of netnography, an ethnography performed online, which has a reduced cost when compared to conventional ethnography and makes it possible to reach a dispersed target audience, such as people with Autism Spectrum Disorder (ASD). Netnography was applied in the Instagram social network to delineate personas of users affected by the TEA and elicit requirements from these personas. We expected to encourage the adoption of netnography in software development due to the reach of the target audience and the variety of possible data to be obtained.
\end{abstract}

Resumo. As fases iniciais do desenvolvimento de software requerem um bom entendimento do público-alvo e suas necessidades. Os métodos convencionais utilizados para alcançar tais objetivos têm um alto custo e os pesquisadores estão sujeitos ao interesse de participação do público-alvo. Este artigo relata a aplicação da netnografia, uma etnografia realizada online, que tem um custo reduzido quando comparada à etnografia convencional e possibilita o alcance de um público-alvo disperso, como é o caso das pessoas com Transtorno do Espectro Autista (TEA). A netnografia foi aplicada na rede social Instagram para delinear personas de usuários afetados pelo TEA e elicitar requisitos a partir destas personas. Espera-se encorajar a adoção de netnografia no desenvolvimento de software tendo em vista o alcance do público-alvo e variedade de dados possíveis de serem obtidos.

\section{Introdução}

Segundo Uchoa e Coutinho (2018), as plataformas online e redes sociais estão promovendo um maior engajamento de participantes externos às equipes de desenvolvimento de software em seus processos. A imersão em comunidades de plataformas online permite a compreensão de opiniões, comportamentos e sentimentos de pessoas em relação a diversas tecnologias (Rodrigues et al., 2018; Fontão et al. 2018; da Silva et al., 2016). O conteúdo disponível e o acesso a pessoas de diferentes contextos nas redes sociais apresentam potencial para o seu uso como fonte de informação e criação de empatia no desenvolvimento de soluções tecnológicas (Marins e Miletto, 2016). 
Neste contexto, a netnografia surge como um processo de imersão em plataformas digitais para investigar determinado público sem altos investimentos, como ocorre com estudos de campo (Melo e Abelheira, 2015). Além disso, a netnografia permite a imersão em comunidades e grupos geograficamente distribuídos, possibilitando a obtenção de dados em maior quantidade e qualidade. Tal fato motivou a adoção da netnografia em um projeto de desenvolvimento de tecnologias acessíveis para pessoas com Transtorno do Espectro Autista (TEA).

A netnografia mostrou-se apropriada para a equipe de desenvolvimento por possibilitar o alcance a mais pessoas envolvidas com o TEA, quando comparado ao número de pessoas disponíveis na região em que o projeto é desenvolvido. Este artigo apresenta um relato de experiência da equipe de desenvolvimento do projeto ProDTeA (Projeto e Desenvolvimento de Tecnologias Acessíveis) na adoção de netnografia para compreensão sobre o público-alvo e suas necessidades. A partir da netnografia, foi possível criar personas para representar diferentes perfis de usuários envolvidos com TEA e que possuem interesse em adotar tecnologias. As personas possibilitaram a identificação de um conjunto inicial de requisitos para tecnologias acessíveis a serem desenvolvidas pelo projeto. Espera-se demonstrar como a netnografia pode ser utilizada como suporte ao desenvolvimento de software.

Este artigo está organizado da seguinte forma: a Seção 2 apresenta a fundamentação teórica. Na Seção 3 são apresentados os trabalhos relacionados. A Seção 4 descreve o relato de experiência. Por fim, na Seção 5 são apresentadas as lições aprendidas e trabalhos futuros.

\section{Fundamentação Teórica}

Nesta seção serão apresentados conceitos necessários para a melhor compreensão do relato de experiência: Transtorno do Espectro Autista, netnografia e personas.

\subsection{Transtorno do Espectro Autista (TEA)}

O Transtorno do Espectro Autista (TEA) é um transtorno do neurodesenvolvimento que tem como principais características o prejuízo na comunicação e interação social, comportamentos repetitivos e padrões restritos de interesses ou atividades (APA, 2014). $\mathrm{O}$ estágio em que o TEA fica evidente varia de acordo com o paciente, geralmente os sintomas estão presentes desde o início da infầncia.

Segundo a APA (2014) há três níveis de gravidade para o TEA: Nível 1 (Exige apoio) - apresentam dificuldades para iniciar interações sociais, respostas atípicas, parecem apresentar interesse reduzido por interações sociais além de inflexibilidade de comportamento e dificuldade em trocar de atividade; Nível 2 (Exige apoio substancial) apresentam déficits graves nas habilidades de comunicação social verbal e não verbal, limitações em dar início a interações sociais e respostas reduzidas ou anormais a aberturas sociais que partem de outros; Nível 3 (Exige apoio muito substancial) apresentam déficits graves nas habilidades de comunicação social verbal e não verbal, grandes limitações em dar início a interações sociais e respostas mínimas a aberturas sociais que partem de terceiros, podem apresentar fala inteligível.

Ainda existe preconceito e falta de informação sobre TEA, principalmente em regiões menos desenvolvidas, como a região em que o projeto ProDTeA é desenvolvido: no município de Russas, no interior do Ceará. Isso ocasiona dificuldade 
em envolver pais, mães e responsáveis em pesquisas de campo, pois muitos preferem não falar sobre o assunto ou não buscam tratamento adequado. Em razão desses fatores, a netnografia apresentou-se como uma alternativa adequada para o projeto, uma vez que nas redes sociais observa-se diversos perfis de pais, mães e profissionais relacionados ao TEA que compartilham experiências diárias.

\subsection{Netnografia}

Segundo Kozinets (2014), a netnografia descreve a aplicação das práticas da etnografia em um ambiente online para a obtenção de informações para uma determinada finalidade. A netnografia pode ser aplicada para levantamento de dados para marketing, pesquisas científicas, obtenção do feedback de usuários e auxílio na criação de produtos. A técnica apresenta vantagens como o baixo custo, alto alcance e a possibilidade de aplicar métodos externos à suas etapas para complementação (Melo e Abelheira, 2015).

Os passos para a execução da netnografia (Kozinets, 2014) são: planejamento, imersão e garantia de preceitos éticos, coleta de dados, análise de dados e obtenção de artefatos requeridos.

Planejamento: definição do foco da pesquisa, artefatos a serem gerados, público-alvo da pesquisa, plataforma online, abordagens para coleta de dados. Entre as abordagens que podem ser efetuadas em um ambiente online pode-se citar: entrevistas, formulários online, chats, fóruns, questionários, videoconferência, brainstorming em grupos online, avaliação de protótipos e observação.

Imersão e garantia de preceitos éticos: ao estabelecer o primeiro contato com o público-alvo, é necessário apresentar-se, explicar a motivação do seu estudo e da sua abordagem e solicitar autorização para a coleta e análise de dados por meio de termo de consentimento livre e esclarecido.

Coleta de dados: coleta manual ou automática de dados por meio das abordagens selecionadas, organização e catalogação para posterior análise.

Análise de dados: após a coleta de dados, os dados obtidos são analisados e organizados para posterior uso na produção dos artefatos.

Obtenção de artefatos requeridos: no contexto de desenvolvimento de software, os dados analisados podem ser usados pela equipe de desenvolvimento de software para delineamento de perfis de usuário, definição de personas, avaliação da experiência do usuário, levantamento de requisitos, entre outros.

\subsection{Personas}

Personas são representações fictícias de um usuário típico de um sistema com informações baseadas em dados coletados do público-alvo (Barbosa, 2010). Cada persona possui os dados pessoais (nome, profissão, idade), perfil (o que gosta de fazer, objetivos), os requisitos que ela precisa para o sistema, as expectativas, habilidades entre outras informações. As informações contidas nas personas dependem do objetivo para o qual está sendo utilizada. Podem ser criadas mais de uma persona para um mesmo objetivo, uma do sexo masculino e uma do sexo feminino, por exemplo.

Ferreira et al. (2018) propõem uma técnica para o desenvolvimento de personas baseado no mapa de empatia, denominada PATHY (Personas EmpATHY). A técnica objetiva criar personas úteis para a identificação de requisitos de software. As principais 
informações que uma persona criada por meio da PATHY possui são: quem, contexto, experiências prévias/preferências, problemas/necessidades/desejos e soluções existentes. Pinheiro et al. (2018) realizaram um estudo experimental aplicando a técnica de proto-persona para a obtenção de requisitos de experiência do usuário. Uma protopersona é um protótipo de persona que une informações sobre o usuário final e o domínio de software, explorando as seguintes informações: dados demográficos, objetivos e necessidades, comportamentos e preferências, dificuldades.

Neste relato, as informações requeridas nas personas PATHY e proto-personas foram integradas para definir os dados de interesse na etapa de coleta de dados.

\section{Trabalhos Relacionados}

\subsection{Adoção de netnografia no contexto de desenvolvimento de software}

Marins e Miletto (2015) relatam o uso de netnografia no ensino de Design Thinking em uma disciplina de empreendedorismo. Os alunos organizados em seis equipes conduziram netnografia utilizando o Facebook, Skype e Whatsapp. Com os dados obtidos, os alunos: (1) construíram mapas de empatia para compreender os usuários; (2) construíram modelos de negócios; e (3) elaboraram protótipos para obter feedback dos usuários que participaram da netnografia. Os autores concluíram que a netnografia configurou uma valiosa fonte de informações aos alunos, que desenvolveram empatia com os usuários. Além disso, a interação entre os alunos e os envolvidos na pesquisa foi um processo colaborativo de aprendizagem, estimulando ideias criativas e inovadoras.

Rodrigues et al. (2018) adotaram a netnografia na avaliação de um aplicativo de monitoramento de controle menstrual. A netnografia foi aplicada em grupos sobre a saúde da mulher no Facebook. Por meio da observação de postagens, as autoras identificaram dados sobre: dúvidas sobre o aplicativo, problemas de interação, indicação de aplicativos, ausência de requisitos, erros de previsão, elogios, entre outros. As autoras afirmaram que a netnografia permitiu obter dados sobre a utilização do aplicativo no contexto de saúde da mulher, enriquecendo a avaliação do ponto de vista da experiência do usuário.

Rotman et al. (2012) levantaram desafios e estratégias para a netnografia efetiva em ambientes online de grande escala. Os autores identificaram cinco desafios: tamanho e escopo do ambiente de pesquisa; unidade de análise; estrutura da interface; conteúdo, ferramentas e instabilidade da plataforma; questões éticas. Como estratégias para tratar esses desafios, os autores apontam o uso combinando de diferentes metodologias, análise estrutural e processamento de linguagem natural.

De forma similar a Marins e Miletto (2015) e Rodrigues et al. (2018), este artigo relata a adoção de netnografia, porém a netnografia foi adotada em um projeto sobre tecnologias acessíveis para pessoas autistas. Assim como Rotman et al. (2012), este relato também permitiu identificar lições aprendidas sobre o uso da netnografia.

\subsection{Uso de plataformas online e redes sociais por pesquisadores e profissionais de Engenharia de Software}

Da Silva et al. (2016) apresentam um processo para análise de papéis de atores e suas relações em um ecossistema de software por meio de uma plataforma online. Por ser uma plataforma de domínio público, a identificação dos atores se deu por meio da 
equipe de desenvolvimento da plataforma e não diretamente na plataforma. A coleta de dados ocorreu por meio entrevistas. Fontão et al. (2017) exploraram a experiência de desenvolvedores no desenvolvimento de aplicativos móveis por meio da mineração de dados sobre questões e respostas postadas no Stack Overflow. Os autores apontam que os resultados podem auxiliar na definição de estratégias para um maior engajamento dos desenvolvedores nos diversos ecossistemas de desenvolvimento mobile. Uchoa e Coutinho (2018) propõem a investigação de estratégias para o desenvolvimento colaborativo de jogos por meio de plataformas digitais. Os autores investigam a integração de crowdsourcing no desenvolvimento e manutenção de jogos digitais.

No contexto de pesquisas sobre TEA, Saha e Agarwal (2015) realizaram um estudo para demonstrar como as comunidades online de autistas se apoiam. Os autores utilizaram a lista de blogs mais influentes disponibilizada pela organização Autism Speaks e analisaram blogs de mães de crianças autistas (13), pais de crianças autistas (10), grupos de autismo (13) e blogueiros autistas (4) entre os anos de 2009 e 2014. Os autores constataram que a comunidade de autistas oferecem um maior suporte, tanto emocional quanto ao compartilhamento de conhecimento em relação a outras comunidades como câncer de mama e transtornos alimentares. Beykikhoshk et al. (2015) realizaram uma pesquisa para investigar como a rede social Twitter poderia ser empregada como fonte de mineração de dados para instruir a população afetada pelo TEA. Os tweets foram coletados e submetidos a pré-processamento, análise de conteúdo e classificação. Os resultados demonstraram que os tweets são ricos em informações sobre a rotina e o autismo, com alto valor informativo.

Os trabalhos mencionados adotam plataformas online e redes sociais para diferentes fins relacionados ao desenvolvimento de software. Este artigo propõe o uso da rede social Instagram em uma pesquisa baseada em netnografia, no contexto de um projeto de desenvolvimento de tecnologias acessíveis para pessoas com TEA.

\section{Aplicação da netnografia no projeto ProDTeA}

Nesta seção serão descritas as etapas adotadas para a aplicação da netnografia no projeto ProDTeA. A Figura 1 ilustra as etapas, que são descritas a seguir.

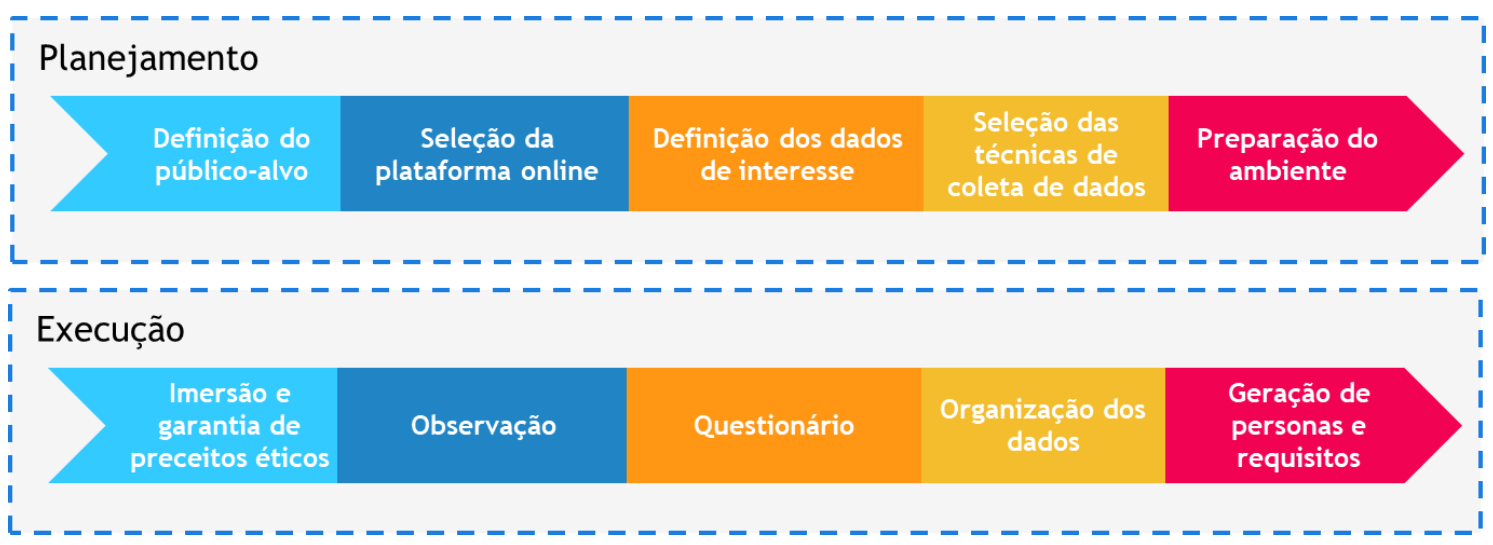

Figura 1. Etapas adotadas na condução da netnografia.

\subsection{Planejamento}

Definição do público-alvo: o projeto ProDTeA visa desenvolver tecnologias acessíveis para pessoas com TEA. Logo, o público-alvo da netnografia consiste em pessoas 
afetadas pelo TEA: pessoas autistas; pais, mães e/ou responsáveis por pessoas com TEA; e profissionais que atuam em intervenções sobre o TEA.

Seleção da plataforma online: o projeto possui uma conta na rede social Instagram, onde são compartilhadas pesquisas e ações desenvolvidas. Observou-se que perfis de pessoas afetadas pelo TEA e instituições passaram a seguir a conta. Com isso, optou-se por conduzir a netnografia no Instagram. Não foram encontrados trabalhos relacionados que aplicassem netnografia com este público-alvo no Instagram.

Definição dos dados de interesse: para os perfis de pais, mães e profissionais relacionados ao TEA, foram estabelecidos como dados de interesse o nome completo, $\mathrm{o}$ gênero, a relação com o autismo, a região, a cidade, o estado, o nome do perfil no Instagram, a escolaridade, a profissão, as dificuldades observadas na interação dos autistas com tecnologias, as melhorias desejadas e se acreditam que a tecnologia pode ser uma boa aliada ao desenvolvimento e aprendizado dos autistas. Para os perfis de autistas, buscou-se identificar a idade, o nível de autismo, se possuía diagnóstico profissional, se possui experiência com tecnologia, quais plataformas (smartphone, tablet, computador...), quais os aplicativos/sites mais utilizados, preferências e atividades que praticam.

Seleção das técnicas de coleta de dados: observação das postagens dos perfis de interesse e aplicação de questionário online para complementar dados não identificados por meio da observação.

Preparação do ambiente: previamente à execução da netnografia, a equipe decidiu: (1) atrair o público-alvo por meio de novas postagens sobre o projeto e (2) buscar e seguir perfis de interesse para a netnografia.

\subsection{Execução}

Imersão e garantia de preceitos éticos: um membro da equipe realizou a abordagem inicial aos perfis de interesse, enviando via direct, uma pequena apresentação sobre o projeto e o objetivo da pesquisa, juntamente com um link para acesso ao Termo de Consentimento Livre e Esclarecido (TCLE) no Google Forms. Caso a pessoa aceitasse participar da pesquisa, deveria indicar no formulário e fornecer dados iniciais: o nome da conta no Instagram, a relação com o autismo e a localização (cidade, estado e região). Esta etapa foi realizada com aproximadamente, 150 perfis de interesse. Obtevese 32 respostas de aceite ao TCLE. Destas, somente 30 puderam ser utilizadas pois duas não foram preenchidas corretamente, não permitindo a identificação do perfil no Instagram. Dos respondentes considerados, 27 pessoas eram mulheres e 3 pessoas eram homens. Sobre sua relação com o autismo (Figura 2), 66.7\% eram mães de autistas, $13.3 \%$ eram profissionais e os demais eram cuidadores, autistas ou pais de autistas. Participaram pessoas de todas as regiões do Brasil, sendo a maioria do Nordeste $(33.3 \%)$ e Sudeste (30\%). Duas pessoas eram brasileiras, mas não residiam no Brasil.

Observação: um membro da equipe elaborou uma ficha de extração para guiar a coleta de dados e validou com a equipe para melhorias. A ficha continha os dados de interesse a serem coletados. Durante a observação, foram consideradas as últimas 50 postagens de cada perfil, além dos stories fixados nos destaques. Ao final da observação, nem todos os campos da ficha de extração foram preenchidos, pois não haviam informações suficientes nas postagens. Para complementá-las, prosseguiu-se para a aplicação de um questionário online. 

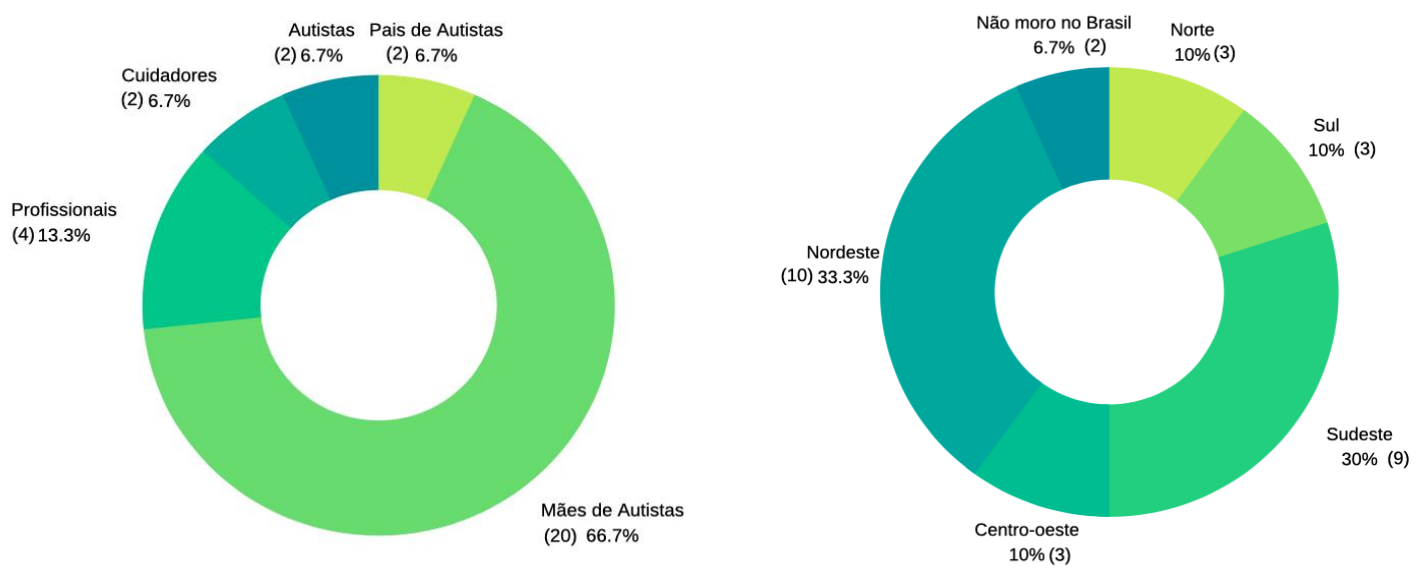

Figura 2. Caracterização dos participantes da netnografia.

Questionário: o link para o questionário online no Google Forms foi enviado por meio do direct para os perfis que haviam consentido com a pesquisa e sido submetidos à observação. $\mathrm{O}$ questionário foi direcionado a extrair dados sobre a experiência do autista com tecnologia, pois este tipo de informação não era comum nas postagens dos perfis. As fichas de extração foram complementadas com as informações coletadas por meio do questionário. Um vídeo de agradecimento foi enviado visando uma aproximação entre o pesquisador e os participantes.

Organização dos dados: as fichas de extração foram separadas em grupos por categoria (pai de autista, mãe de autista, cuidador(a), profissional e autista) no intuito de facilitar a criação das personas.

Geração de personas: os dados das fichas de extração foram a base para geração de personas. O nome e sobrenome das personas são fictícios e não condizem com nenhum dos participantes da pesquisa. A Figura 3 mostra um mapeamento dos perfis cujas fichas de extração foram utilizadas para a geração de cada persona.

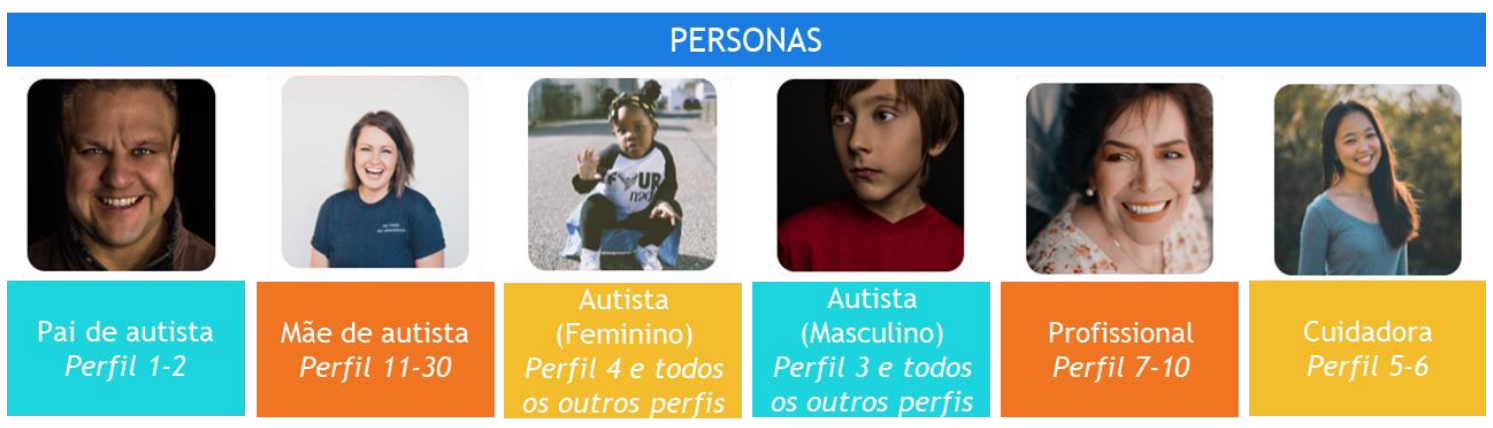

Figura 3. Mapeamento de perfis geradores de cada persona.

As técnicas PATHY e proto-personas foram adotadas como base para a definição das informações das personas. As personas possuem três campos: (i) perfil, (ii) biografia e (iii) autismo e tecnologia. O campo "perfil" foi baseado nos campos "quem" da PATHY e "dados demográficos" da proto-persona; o campo "autismo e tecnologia" foi baseado nos campos "comportamentos e preferências", "objetivos e necessidades" e "dificuldades" da proto-persona e nos campos "experiências prévias/preferências" e "problemas/necessidades/desejos" da PATHY. O campo "biografia" apresenta uma breve descrição da persona, com informações retiradas da 
observação. As imagens utilizadas nas personas são de uma plataforma de fotos livres de direitos autorais chamada Unsplash ${ }^{1}$ e as personas foram criadas utilizando a ferramenta Canva ${ }^{2}$.

Para gerar a persona da mãe de um autista (Figura 4) foram analisadas as fichas de extração de 20 perfis de mãe. A persona criada foi nomeada como Fabiana Freitas, mãe de duas crianças autistas. Fabiana incentiva seus filhos a usarem tecnologia, em especial jogos que possam auxiliar no aprendizado e alfabetização. Para Fabiana, recursos de personalização são importantes nas tecnologias, assim como a portabilidade para serem acessíveis em diferentes ambientes. Para gerar a persona da autista do sexo feminino (Figura 4) foi analisada a ficha de extração da autista do sexo feminino e todas as outras fichas de extração dos perfis de pessoas relacionadas ao autismo. Yasmin Rebouças é uma criança diagnosticada com autismo nível 2, que exige apoio substancial para suas atividades diárias. Por isso, Yasmin tem o acompanhamento de uma psicopedagoga e uma cuidadora. Sua mãe vem incentivando a inclusão de tecnologia em sua rotina, especialmente jogos educativos. Yasmin gosta da variedade de cores e recursos audiovisuais dos aplicativos que utiliza.
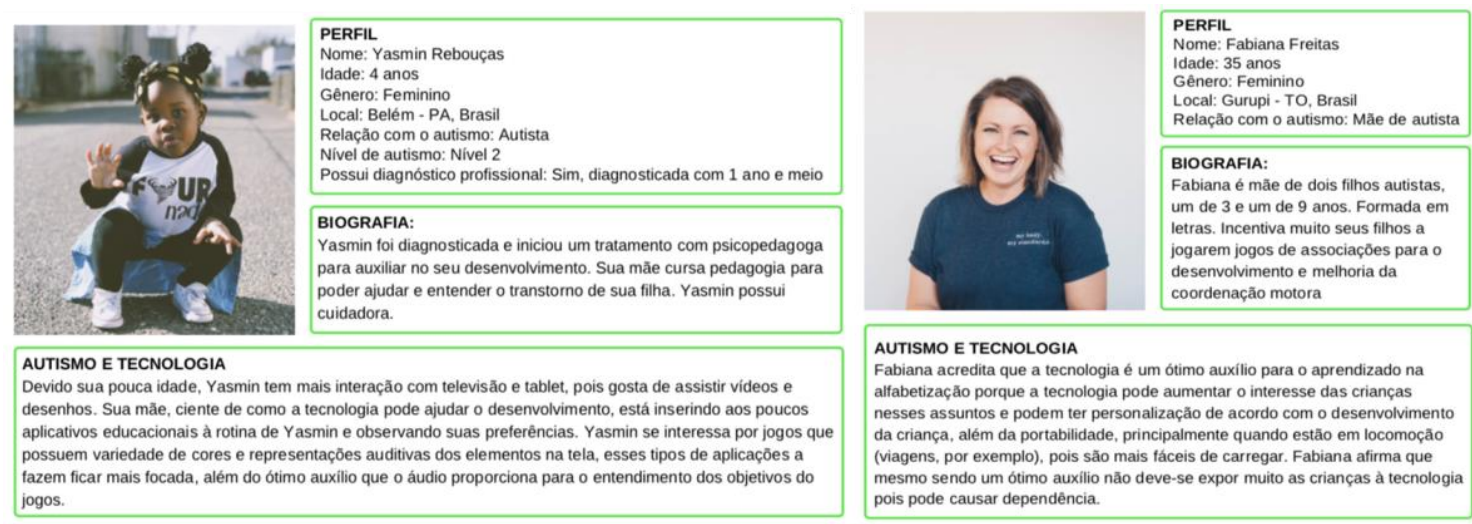

Figura 4. Exemplos de personas geradas por meio da netnografia.

Extração de requisitos: para extrair os requisitos, foi utilizado o campo "autismo e tecnologia" das personas, pois continha as necessidades, dificuldades, melhorias esperadas e preferências do uso de tecnologia pelos autistas. A Figura 5 ilustra o mapeamento do campo das personas utilizado para elicitar os requisitos. Os requisitos descrevem funcionalidades podem ser implementadas em qualquer sistema com foco em autismo, como jogos, aplicativos educacionais e podem ser incorporadas a sites para que sejam acessíveis para usuários com TEA. O conjunto completo de personas e requisitos iniciais obtidos pode ser consultado em Melo (2019).

\section{Conclusões e Lições aprendidas}

Este artigo apresentou um relato de experiência da condução de netnografia no projeto ProDTeA, que visa desenvolver tecnologias acessíveis para pessoas com TEA. A netnografia possibilitou a geração de personas e requisitos para futuras aplicações. Como lições aprendidas, podemos destacar que:

\footnotetext{
${ }^{1}$ https://unsplash.com

${ }^{2}$ https://www.canva.com
} 


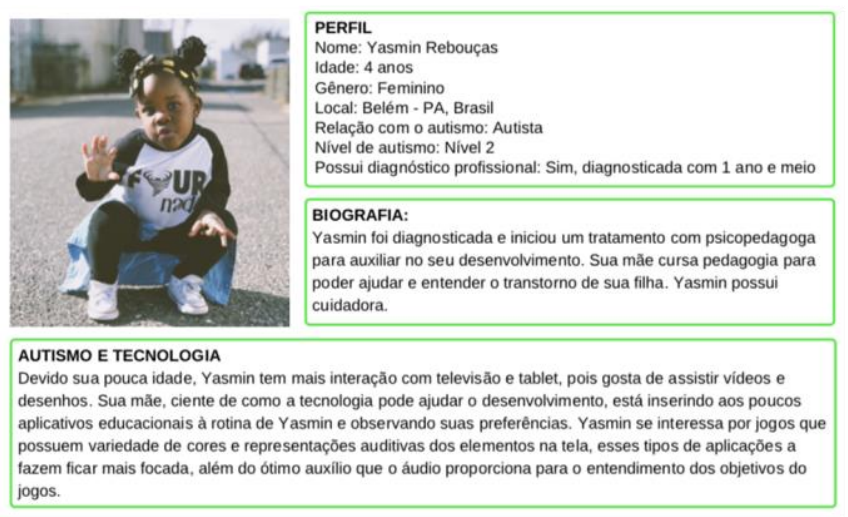

\begin{tabular}{|l|l|}
\hline Identificador & 002 \\
\hline Tipo de requisito & Funcional \\
\hline $\begin{array}{l}\text { Nome do } \\
\text { Requisito }\end{array}$ & Conter instruções de manuseio \\
\hline Descrição & $\begin{array}{l}\text { O sistema deve conter instruções de manuseio para } \\
\text { um melhor entendimento de suas funcionalidades e } \\
\text { facilitar o aprendizado dos autistas. }\end{array}$ \\
\hline Fonte & Adriana Rodrigues, Yasmin Rebouças \\
\hline Identificador & 003 \\
\hline Tipo de requisito & Funcional \\
\hline $\begin{array}{l}\text { Nome do } \\
\text { Requisito }\end{array}$ & Instruções por áudio \\
\hline Descrição & $\begin{array}{l}\text { O sistema deve possuir instruções por áudio para que } \\
\text { crianças que ainda não saibam ler ou estão em fase } \\
\text { de aprendizado possam entender seu funcionamento. }\end{array}$ \\
\hline Fonte & Yasmin Rebouças \\
\hline
\end{tabular}

Figura 5. Exemplo de requisitos extraídos a partir das personas.

1) A rede social Instagram é uma fonte rica de informações e dados para netnografia, pois seus usuários compartilham um grande volume de informações e o direct permite a interação entre o pesquisador e os participantes.

2) Algumas particularidades do Instagram dificultaram a condução da netnografia: (a) o algoritmo para geração do feed de publicações não é baseado em ordem cronológica e mostra primeiramente as publicações de perfis de interesse, o que limitava as visualizações das postagens do perfil do projeto; (b) o Instagram bloqueou o perfil do projeto algumas vezes por seguir perfis com a mesma temática, sendo necessário um tempo de espera para continuar a imersão; (3) o Instagram bloqueou o início de conversas pelo direct, atrasando o envio dos TCLES e dos questionários.

3) As postagens no Instagram possuem uma grande diversidade de conteúdo, porém por meio da observação, foram obtidas poucas informações sobre o uso de tecnologia por pessoas autistas. Talvez plataformas mais direcionadas a esta temática possibilitem resultados mais ricos e direcionados ao uso de tecnologia.

Ainda assim, a netnografia possibilitou uma maior imersão da equipe em relação ao público-alvo, indicando que a netnografia é uma técnica com potencial para ser adotada em projetos com público-alvo pouco acessível ou disperso. Espera-se encorajar a condução de netnografia em outras plataformas online e redes sociais, com diferentes públicos, para geração de diferentes artefatos de software. Pretende-se investigar o ponto de vista da equipe de desenvolvimento sobre o impacto da netnografia como suporte ao desenvolvimento de software e validar a qualidade das personas criadas com profissionais da área de desenvolvimento e TEA.

\section{Referências}

American Psychiatric Association. (2014) Manual Diagnóstico Estatístico de Transtornos Mentais: DSM-5. Porto Alegre: Artmed. 2014.

Barbosa, S. D. J. (2010) Interação Humano-Computador. Rio de Janeiro: Elsevier, 2010.

Beykikhosh, A., Arandjelović, O., Phung, D., Venkatesh, S., e Caelli, T. (2015) Using Twitter to Learn about the Autism Community in Soc. Netw. Anal. Min 2015. Springer, 2015, p. 5-22. 
da Silva, R., Aguiar, L., dos Santos, R., \& Gengivir, E. (2016). Levantamento de Papéis e Atores em um Ecossistema de Software no Domínio Público. In Anais do I Workshop sobre Aspectos Sociais, Humanos e Econômicos de Software, (pp. 76-80).

Ferreira, B., Barbosa, S., \& Conte, T. (2018). Creating Personas focused on Representing Potential Requirements to Support the Design of Applications. In Proc. of the 17th Brazilian Symposium on Human Factors in Computing Systems (pp. 19).

Fontão, A., Ekwoge, O., Santos, R., \& Dias-Neto, A. (2017). Facing up the primary emotions in Mobile Software Ecosystems from Developer Experience. In Anais do II Workshop sobre Aspectos Sociais, Humanos e Econômicos de Software, (pp. 5-11).

Kozinets, R. V. (2014) Netnografia [recurso eletrônico]: Realizando pesquisa etnográfica online. Porto Alegre: Penso, 2014.

Marins, M., Miletto, E. M. (2015) Uso das Redes e Aplicações Multiplataformas como Fonte de Empatia e Criatividade do Design Thinking na Disciplina de Empreendedorismo, no Ensino Superior. In Anais do Computer on the Beach, (pp. 394-403).

Melo, G.A.D.F. (2019). Investigando o uso da netnografia como meio de levantamento de dados para a geração de personas e elicitação de requisitos para sistemas com foco em pessoas com transtorno do espectro autista: um estudo de caso. Trabalho de Conclusão de Curso (Graduação em Engenharia de Software) - Universidade Federal do Ceará, Campus de Russas, Russas, 2019. Disponível em: http://www.repositorio.ufc.br/handle/riufc/49697

Melo, A. \& Abelheira, R. (2015) Design Thinking \& Thinking Design. São Paulo, Novatec.

Pinheiro, E. G., Lopes, L. A., Conte, T. U., \& Zaina, L. A. M. (2018). The contribution of non-technical stakeholders on the specification of UX requirements: an experimental study using the proto-persona technique. In Proceedings of the XXXII Brazilian Symposium on Software Engineering (pp. 92-101).

Rodrigues, K., Salerno, L., Johnstone, L., Jesus, M., \& Silveira, M. (2018). A Saúde da Mulher e a Tecnologia: Uma Análise Heurística e Netnográfica do Aplicativo Clue. In Anais Estendidos do XVII Simpósio Brasileiro sobre Fatores Humanos em Sistemas Computacionais (pp. 1-10) doi:10.5753/ihc.2018.4202.

Rotman, D., Preece, J., He, Y., Druin, A. (2014) Extreme Ethnography: Challenges for Research in Large Scale Online Environments, in Proceedings of the 2012 iConference, ACM, 2012, p. 207-2014.

Saha, A., \& Agarwal, N. (2015). Demonstrating social support from autism bloggers community on twitter. In Proceedings of IEEE/ACM International Conference on Advances in Social Networks Analysis and Mining (ASONAM) (pp. 1053-1056).

Uchoa, A., \& Coutinho, E. (2018). De que Forma a Cultura do Compartilhamento e Modificação pode Colaborar no Processo de Desenvolvimento de Jogos?. In Anais do III Workshop sobre Aspectos Sociais, Humanos e Econômicos de Software (pp. 1-5) doi:10.5753/washes.2018.3475 